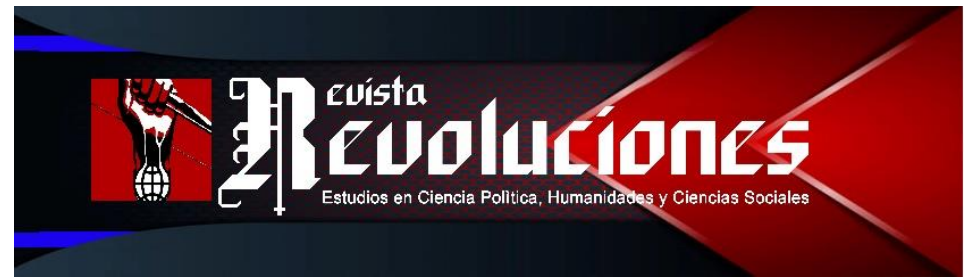

\title{
EL CICLO DE LA LANA: EXPORTACIÓN, CAMPESINOS Y CONFLICTO SOCIAL EN PUNO DE 1850 A 1930
}

\author{
The wool cycle: export, peasants and social conflict in Puno from 1850 to \\ 1930
}

\author{
Carlos Guillermo Carcelén'1 \\ UNIVERSIDAD NACIONAL MAYOR DE SAN MARCOS \\ PERÚ \\ ccarcelenr@unmsm.edu.pe \\ https://orcid.org/oooo-0001-7645-4955
}

\author{
Luis Daniel Morán Ramos ${ }^{2}$ \\ UNIVERSIDAD NACIONAL MAYOR DE SAN MARCOS \\ PERÚ \\ lmoranr@unmsm.edu.pe \\ https://orcid.org/o0oo-0002-8244-5390
}

DOI: https://doi.org/10.35622/j.rr.2021.04.005

Recibido: 20-V-2021 / Aceptado: 21-VI-2021 / Actualizado: 25-VI-2021

\section{Resumen}

La exportación de lana fue por mucho tiempo, para el sur peruano la principal actividad, ya que, teniendo como objetivo a la exportación, se organizó la producción, el mercado y los mecanismos de comercialización de la lana, tanto de alpaca y oveja. Dando lugar con esto a la conformación de una sociedad en la cual la producción y comercialización de lana, creará y recreará las características propias de la vida y relaciones sociales en un espacio geográfico importante por su extensión. Esta actividad presentará diversos conflictos entre los productores directos que fueron las comunidades campesinas, los comercializadores y los

\footnotetext{
${ }^{1}$ Doctor en Historia Ambiental y del Clima adquirida en España y Francia, con investigaciones sobre las respuestas culturales y sociales a los cambios ambientales y los desastres naturales desarrolladas en instituciones de la Cooperación Internacional como el Programa Hidrológico Internacional de la Unesco, el Instituto Francés de Estudios Andinos y el Instituto de Investigaciones para el Desarrollo de la cooperación francesa. Investigaciones que fueron presentadas en Conferencias Magistrales y Seminarios especializados internacionales, y publicadas en revistas científicas peruanas y de varios países como Argentina, Bolivia, Chile, Colombia, España, Estados Unidos, Francia, México, entre otros. Consultor del Programa Especial MECEP - Banco Interamericano de Desarrollo en el Área de Promoción de Innovaciones Educativas del Ministerio de Educación. Con experiencia en gestión editorial, edición y corrección de textos en editoriales especializadas en investigación en Ciencias Sociales y Humanidades, así como evaluador de artículos en revistas especializadas de varios países latinoamericanos. Con experiencia de más de veinte años en la docencia universitaria en las facultades de Ciencias Sociales y de Educación de la Universidad Nacional Mayor de San Marcos.

2 Docente Investigador de la Facultad de Educación en la Universidad San Ignacio de Loyola y Docente de Historia en la Facultad de Ciencias Sociales en la Universidad Nacional Mayor de San Marcos (Perú). Actualmente Investigador Renacyt (Carlos Monge, Nivel III) en CONCYTEC-Perú. Además, coordinador del Grupo de investigación Educación y Sociedad en el Perú y América Latina en la Facultad de Educación de USIL. Ha sido becario Roberto Carri (2009-2010) y becario doctoral del Instituto Ravignani de la UBA por el CONICET-Argentina (2011-2016). Doctor en Historia en la Universidad de Buenos Aires (Argentina), Magister en Historia en el Instituto de Altos Estudios Sociales de la Universidad Nacional de San Martín (Argentina) y Licenciado en Historia en la Universidad Nacional Mayor de San Marcos (Perú).
}

$$
\text { ISSN: 2710-0499 ISSN-L: 2710-0480 }
$$

Esta obra está bajo una licencia internacional Creative Commons Atribución 4.o. 
terratenientes, tanto por la posesión de la tierra o el control de las redes comerciales regionales para abastecer a la demanda internacional.

Palabras Clave: Exportación, lana, campesinado, conflicto social, Puno.

\begin{abstract}
The export of wool was for a long time, for the south of Peru the main activity, since with the objective of exporting, the production, the market and the marketing mechanisms of wool, both alpaca and sheep, were organized. With this, giving rise to the formation of a society in which the production and commercialization of wool will create and recreate the characteristics of life and social relations in a geographical space that is important due to its extension. This activity will present various conflicts between the direct producers that were the peasant communities, the traders and the landowners, both for the possession of the land or the control of the regional commercial networks to supply the international demand.
\end{abstract}

Keyword: Export, wool, peasantry, social conflict, Puno.

\title{
INTRODUCCIÓN
}

Para el estudio de los problemas del campesinado en cuanto a la producción ganadera de camélidos en los Andes tenemos el balance bibliográfico de Flores Ochoa (1983), mientras que los problemas socioeconómicos que planteó la distribución y la articulación de la producción de lana altiplánica en el mercado internacional desde el siglo XIX son vistos de manera histórica desde la década de 1970, es por ello que aquí asumimos la tarea de mostrar en un marco más amplio los problemas que acarreó la comercialización de la lana altiplánica.

Con respecto a esto, existe una serie de investigaciones que nos permiten tratar de explicar la dinámica de la "Actividad Lanera”, entre las más importantes tenemos a las de Heraclio Bonilla (1974 y 1975), Alberto Flores Galindo (1977), Geoff Bertram (1977), Gordon Appleby (1979), Nils Jacobsen (1983, 1983b, 1993 y 2013), Manuel Burga y Wilson Reátegui (1981), Manuel Burga y Alberto Flores Galindo (1987 (1980)), entre otros.

La importancia económica social y política de la "actividad lanera” en el Sur Andino a lo largo de la historia, puede ser entendida con la siguiente cita:

“...Secularmente, ésta región del Perú fue uno de los ejes económicos que articuló el espacio Peruano colonial, puesto que en ella estuvo ubicado Potosí y el complejo minero... Al quebrarse el monopolio español, al abrir la Independencia de los Puertos del Perú a todas las potencias del mundo, al ingresar la economía peruana dentro del espacio de control y de dominio británico, requerir las industrias textiles de ésta potencia nuevas fuentes para el aprovisionamiento de materias primas, una vez más el sur peruano, en función de sus recursos, fue forzado a una nueva especialización. Esta vez la economía del sur peruano giró en torno a la explotación y exportación de las lanas de alpaca y de vicuña, principalmente, y las de oveja" (Bonilla, 1974, p. 32).

Revista Revoluciones -69- Vol. 3, No 4 (2021), pp. 68-85

Esta obra está bajo una licencia internacional Creative Commons Atribución 4.0, 
La exportación de Lana de plantea, así como una actividad económica, que se inicia con las comunidades campesinas, pequeñas estancias y algunas haciendas del Altiplano; y que pasa por una serie de intermediarios, que tanto al "menudeo" o como "mayoristas", abastecerán a las grandes casas comerciales exportadoras.

La comercialización de la lana, tanto de alpaca, llama y oveja, se constituirá en un sistema organizado desde la misma producción hasta la exportación. En éste sentido desde fines del siglo XIX, hasta la primera década del presente siglo se observa un esquema más o menos permanente, compuesto, como señalan Burga y Flores Galindo (1987(1980)) y Burga y Reátegui (1981), por:

1.- "Productor", sea comunero o hacendado;

2.- "Alcanzador", que acopia pequeñas cantidades en su zona de origen, por lo general era un productor comunero, o un pariente del "rescatista";

3.- "Rescatista", era el pequeño o mediano comerciante del interior que cambiaba productos o compraba las lanas a los productores o a los alcanzadores; sea individualmente o en las ferias;

4.- "Agente o Sucursal", era un comerciante mayorista que se encargaba de comprar grandes cantidades de lana por encargo de las grandes "casas comerciales";

5.- "Casa comercial Arequipeña”, se encargaba del gran acopio y embarque de la lana con rumbo a los mercados internacionales para cubrir la demanda de la industria textil.

La importancia económica de la Lana, puede traducirse también en las cifras, sobre todo con las relacionadas con la exportación. Entre los años 1854 y 1919, la lana de alpaca y llama representaron aproximadamente el $10.78 \%$ en promedio del total de las exportaciones a Gran Bretaña. Mientras que la lana de oveja representó aproximadamente el $4.49 \%$ en promedio de las exportaciones al mismo reino, durante los mismos años. Mientras que entre 1900 y 1930, la lana de las tres especies representó aproximadamente $5 \%$ en promedio total de los productos exportados, fundamentalmente a la Gran Bretaña y EEUU.

\begin{tabular}{|c|c|c|c|c|c|c|c|}
\hline \multirow{3}{*}{ AÑOS } & \multicolumn{5}{|c|}{$\begin{array}{l}\text { Cuadro } \mathrm{N}^{\circ} 1 \\
\text { de exportación } 1900 \text { - } 1930 \\
\text { rcentuales sobre el total) }\end{array}$} & & \\
\hline & & & PR & UCTO & & & \\
\hline & Azúcar & Algodón & Caucho & Lana & Petróleo & Cobre & Otros \\
\hline 1900 & 32 & 7 & - & 7 & - & 14 & 40 \\
\hline 1905 & 32 & 7 & 16 & 8 & - & 10 & 27 \\
\hline 1910 & 20 & 14 & 18 & 7 & 2 & 13 & 26 \\
\hline 1915 & 26 & 11 & 5 & 5 & 10 & 29 & 14 \\
\hline 1920 & 42 & 30 & 1 & 2 & 5 & 12 & 8 \\
\hline
\end{tabular}

Revista Revoluciones $=70$ - Vol. 3, N 4 (2021), pp. 68-85

Esta obra está bajo una licencia internacional Creative Commons Atribución 4.0. 


\begin{tabular}{|l|l|l|l|l|l|l|l|}
\hline 1925 & 11 & 32 & 1 & 4 & 24 & 18 & 10 \\
\hline 1928 & 13 & 21 & - & 4 & 28 & 20 & 14 \\
\hline 1930 & 11 & 18 & - & 3 & 30 & 19 & 19 \\
\hline
\end{tabular}

Fuente: Burga y Flores Galindo (1987; 1980, p. 73).

\section{DESARROLLO}

\section{Exportaciones de lana}

La exportación de la lana, se constituyó en un rubro importante para la economía del Perú y en especial en la del Sur. La importancia de la lana puede observarse con claridad en los niveles de exportación a Gran Bretaña, entre los años 1854 a 1919. Durante estos años podemos observar el crecimiento y disminución de la exportación de los distintos tipos de lana.

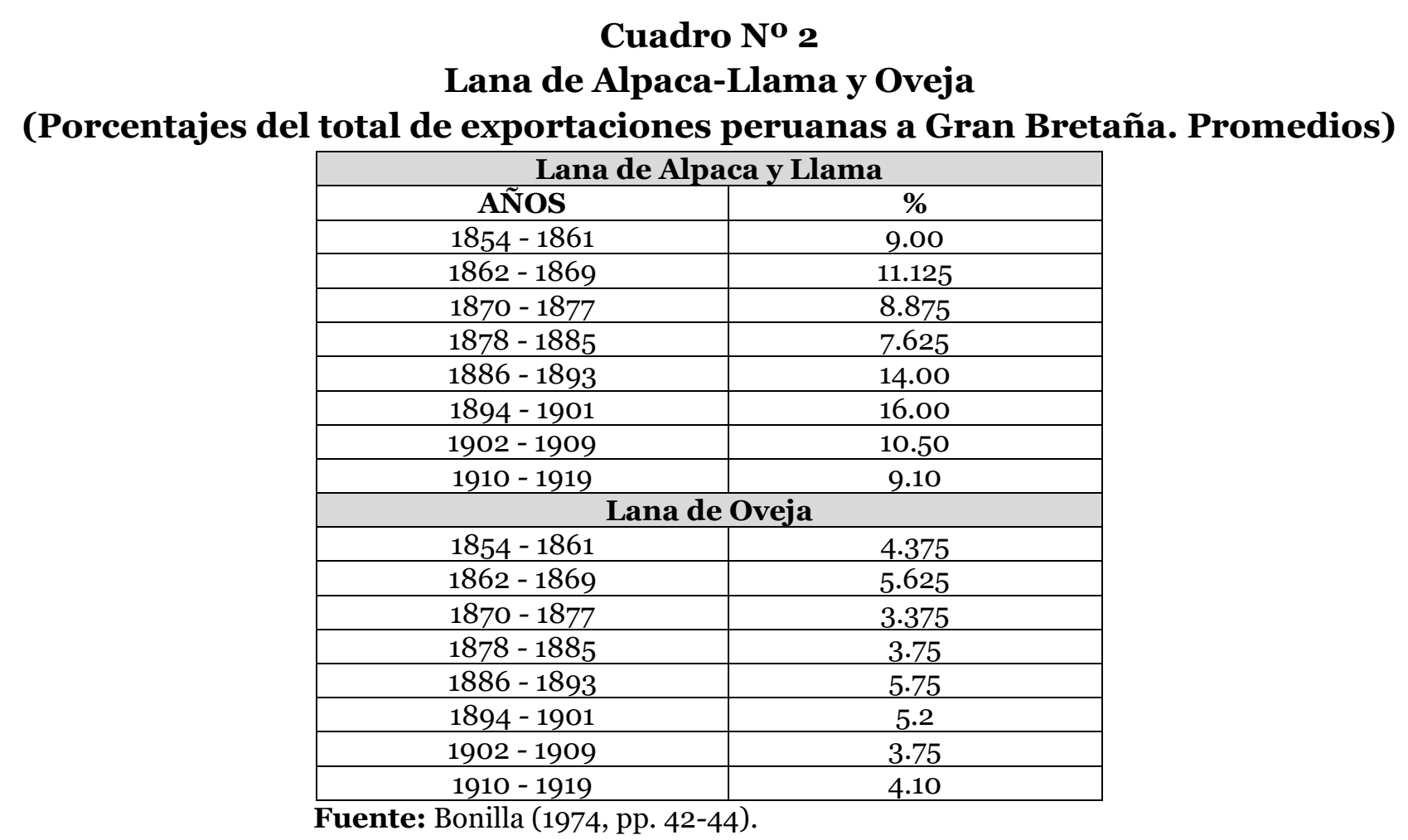

La exportación de lana tuvo fluctuaciones de acuerdo a la demanda, tanto que en la Primera Guerra Mundial, la demanda alcanzó niveles nunca vistos en Europa, con una consecuente elevación de los precios. La lana de oveja paso de 21.2 peniques por kilo en 1913 a 34.2 en 1916 y llegó a un pico de 79.4 peniques en 1918. Durante 1919 se mantuvo alto, a 70.1, pero en 1920 y 21 se produjo un rápido declive pagándose 19.8 peniques por kilo en los puertos británicos.

El precio de 1918 estuvo un $137 \%$ por encima del precio más alto pagado por lana de ovino entre 1855 y 1914 (33.5 peniques por kg. en 1872). Mientras que el volumen de las 
exportaciones de lana de Mollendo, no aumentaron tan rápido como los precios. De 1,207 toneladas métricas en 1913 aumentó 1,905 en 1915 y alcanzó 2,560 como pico, manteniéndose durante 1918 y 19, luego bajando bruscamente a 576 toneladas en 1921, el nivel más bajo alcanzado desde 1855. El valor total de las exportaciones en 1918 fue superior a las 700 mil libras esterlinas, representa un incremento de $211 \%$ por encima del pico anterior correspondiente al período 1855-1914, alcanzando en 1864 un aproximado de 225 mil libras esterlinas.

El "boom" de las exportaciones de lana de alpaca fue menos espectacular debido principalmente a un incremento más débil en los precios. Para sustentar esto utilizamos los cuadros de las exportaciones de lana elaborados por Nils Jacobsen, con respecto a los puertos de Islay Mollendo. De acuerdo con Jacobsen este "boom" de los años 1914-19, puede ser entendido "dependiendo en cierta medida del criterio de evaluación empleado" (Jacobsen, 1983, p. 93). Ya que para unos "las exportaciones laneras se mantuvieron relativamente constantes" (Spalding 1977, p. 30), y para otros la economía de la región era "más o menos languideciente" (Chevalier, 1966, p. 821).

Pero hay otros investigadores que encuentran en las estadísticas de exportación lanera del sur peruano una tendencia de crecimiento a largo plazo. Aquí se incluyen a Geoff Bertram (1977), Gordon Appleby (1979), Alberto Flores Galindo (1977), Manuel Burga y Alberto Flores Galindo (1987(1980)) y Heraclio Bonilla (1974).

Este último elaboró un esquema de ciclos para las exportaciones laneras del sur peruano dentro de una tendencia de expansión de largo plazo. Basándonos en el volumen de las exportaciones desarrollamos una periodificación en las que las fluctuaciones cíclicas forman parte de una tendencia de expansión a largo plazo, desde la Independencia del Perú.

Cuadro No 3
Lana de Ovino, Alpaca y Llama.
\begin{tabular}{|c|c|}
\hline \multicolumn{2}{|c|}{ Lana de Ovino } \\
\hline AÑOS & PERÍODOS \\
\hline $1823-1840$ & Crecimiento \\
\hline $1840-1852$ & Declive \\
\hline $1852-1864$ & Crecimiento \\
\hline $1864-1880$ & Declive \\
\hline $1880-1913$ & Crecimiento \\
\hline Lana de Alpaca y Llama \\
\hline $1839-1876$ & Crecimiento \\
\hline $1876-1880$ & Declive \\
\hline $1880-1886$ & Recuperación \\
\hline $1886-1919$ & Estabilidad \\
\hline
\end{tabular}

Fuente: Bonilla (1974, p. 35) y Jacobsen (1983b, p. 97).

Ante esto se plantea también necesario establecer una diferencia entre el desarrollo de los precios internacionales para las lanas peruanas y las fluctuaciones de los volúmenes de exportación, ya que así podemos observar la elasticidad de la demanda e incluso de la 
producción total de las lanas, por lo menos de la parte que se exporto. Los precios que se pagaron por la lana de ovino peruano en los puertos británicos, sugieren el siguiente ritmo en la demanda de la industria textil:

\section{Cuadro No 4 \\ Lana de Ovino y Alpaca}

\begin{tabular}{|c|c|}
\hline & \multicolumn{2}{|c|}{ Lana de Ovino } \\
\hline AÑOS & \multicolumn{2}{c|}{ PRECIOS } \\
\hline 1855 (o antes)-1872 & Precios en ascenso (con una fuerte caída entre 1867-1870) \\
\hline $1872-1897$ & Precios en descenso \\
\hline $1897-1918 / 19$ & Precios en ascenso (con un período de estancamiento entre \\
& Lana de alpaca \\
\hline \multicolumn{2}{|c|}{ Lan) } \\
\hline 1855(o antes)-1864 & Precios en ascenso \\
\hline $1864-1888$ & Precios en descenso \\
\hline $1888-1914$ & Precios estancados \\
\hline $1914-1918 / 19$ & Precios en ascenso \\
\hline
\end{tabular}

Fuente: Jacobsen (1983b, p. 98).

Mientras que las tendencias a largo plazo en el volumen de exportaciones laneras procedentes del sur peruano nos muestran lo siguiente:

Cuadro $\mathrm{N}^{\circ} 5$

Lana de Ovino y Alpaca

\begin{tabular}{|c|c|}
\hline \multicolumn{2}{|c|}{ Ovino } \\
\hline AÑOS & PERÍODOS \\
\hline 1855 (o antes)-1867 & Crecimiento \\
\hline $1867-1882$ & Declive \\
\hline $1882-1892$ & Recuperación \\
\hline $1892-1908$ & Estancamiento \\
\hline $1908-19 l 7 / 18$ & Crecimiento \\
\hline \multicolumn{2}{|c|}{ Alpaca } \\
\hline 1855 (o antes)-1873/76 & Crecimiento \\
\hline $1873 / 76-1883$ & Declive \\
\hline $1883-1905 / 06$ & Crecimiento \\
\hline $1905 / 06-1914$ & Estancamiento \\
\hline $1914-1918$ & Crecimiento \\
\hline
\end{tabular}

Fuente: Jacobsen (1983b, p. 99).

Este crecimiento se refleja en los precios, ya que estos tuvieron un ritmo acorde con una demanda en aumento, que responde a las tendencias de crecimiento de los países industrializados, lo que se aprecia con claridad en las fluctuaciones de los precios de la lana de oveja, mientras en la de alpaca hay cierta disparidad, que va ligada a la variable mercado de moda, entre otras.

Jacobsen diferencia su interpretación de los ritmos de exportación, con la de Bonilla; primero por la periodificación, ya que Bonilla utiliza promedios móviles para siete años; pero la sustancial diferencia está en la interpretación de las exportaciones de lana de alpaca desde la 
ISSN: 2710-0499 ISSN-L: 2710-0480

segunda mitad de la década de 1880, ya que Bonilla sólo utiliza las fuentes británicas y no toma en consideración la creciente demanda norteamericana.

Tanto Jacobsen y Bonilla concuerdan en que "no se mantiene una sincronización perfecta entre el ritmo de la economía internacional y las exportaciones laneras del sur peruano durante la segunda mitad del siglo XIX y las primeras décadas del siglo XX". Pero Jacobsen mantiene discrepancias que se explican por la influencia de factores exógenos y endógenos; y además durante el Período comprendido entre 1855 y 1920 se aprecian dos fases, "que deben considerarse en especial debido al desarrollo divergente de los precios y volúmenes de exportación, los años de 1867-1872 y 1882-1897" (Jacobsen, 1983b, pp. 99-100).

La primera muestra que tanto volumen exportado y precios descienden (1886-67 a 1870), luego los precios alcanzan un pico en 1872, mientras que los volúmenes apenas se recuperan. La segunda se explica por factores exógenos (Guerra contra Chile) y sus consecuencias. Los volúmenes de lana de ovino bajaron en 1882 a un $55 \%$ del volumen promedio para los años 1855-59. Los precios bajaron, pero con la estabilización política, los exportadores incrementaron los volúmenes, pero los precios siguieron cayendo hasta inicios de la década de 1890. Para esta situación son importantes los informes de los Cónsules Británicos, sobretodo del Cónsul Robilliard entre los años 1880 y 1886 (Bonilla 1975, pp. 5-9).

Tanto la recuperación de la primera y la segunda fase son explicadas por el incremento de la demanda internacional que se traduce en un incremento de precios, dando un incentivo para el incremento extensivo (por lo general) de la producción de lana de ovino. Estas dos fases son épocas extremas que se aprecian en todo un proceso que presenta una tendencia al crecimiento; el cual se aprecia en un incremento de los volúmenes exportados y de los precios a largo plazo.

Como se observó en esas dos fases los factores exógenos son esenciales para el crecimiento de la actividad ganadera del sur. Pero también se aprecian factores endógenos, de diversa índole, como alteraciones políticas y militares, desastres climatológicos y las epidemias en los animales, que hicieron que el volumen de las exportaciones fuese totalmente insensible a los precios, los cuales se elevaron por la demanda internacional, pero debido a estos factores endógenos, no se aprecia una correlación entre el aumento de precios y de volúmenes exportados.

En lo referente a la lana de alpaca, "la correlación entre demanda internacional y volumen de exportación es mucho más débil que en el caso de la lana de ovino" (Jacobsen, 1983b, p. 101), y además que el incremento de las exportaciones durante 1855-1918, presenta interrupciones relativamente breves. Este autor explica este crecimiento por las condiciones sociales y políticas bajo las cuales se producía y comercializaba la lana de alpaca; como que la mayoría de la producción de esta lana provenía de los campesinos indígenas; también influenciada en este crecimiento sostenido el crecimiento a largo plazo de la población animal. A esto se aúna la importancia de la producción peruana, por sus ventajas naturales para la cría de los camélidos, en la zona alto andina. 
De acuerdo con Jacobsen las exportaciones de las lanas del sur peruano vario considerablemente de acuerdo al tipo de la lana. La lana de alpaca tuvo una tendencia de crecimiento para todo el período 1855-1918, mientras que la de ovino presenta ciclos, los cuales tienen una relación con la segunda onda larga de Kondratieff que refleja las fluctuaciones en el crecimiento de la de los países industrializados. El ritmo de crecimiento de la exportación de la lana de ovino, es un reflejo de esta onda larga, con adaptaciones específicas a las condiciones políticas y económicas que prevalecían en el sur del Perú, es decir que este ritmo de crecimiento visto en una onda larga solo es explicado cuando se observa la relación entre los factores endógenos y exógenos, que confluyen en una región y actividad específica.

\section{Sobre la distribución de la propiedad}

Jacobsen realizó un análisis cuantitativo de los contratos notariales relacionados con la propiedad rural en una provincia del Altiplano, Azángaro, desde la segunda mitad del siglo XIX y las dos primeras décadas del presente siglo. Se observa que hay un ritmo creciente de ventas de tierra y tiene un pico a mediados de la década de 1860 y luego hay una aceleración a partir de la década de 1890, con un pico que se produce durante la Primera Guerra Mundial. Entre 1851 y 1910 hay 3,06o contratos de venta, mientras que durante 1911 y 1920 hay entre 1,500 y 1,700 transacciones (Jacobsen, 1983b, p. 103).

Del total de transacciones se puede apreciar previa desagregación de acuerdo a los antecedentes sociales de vendedores y compradores; que el mercado de tierras tiene naturaleza cíclica. Del total, 1,646 transacciones fueron ventas de campesinos indígenas a grandes propietarios hispanizados. Para la década de 1860 el 30\% de la tierra vendida pasaba de campesinos indígenas a grandes propietarios hispanizados. Entre 1901 y 1910 alcanzo el $64 \%$ sobre el total de contratos de ventas efectuados durante el período de 1851-1910.

Este boom de transferencias de tierras, llamado también por una usurpación de tierras de comunidad, alcanzó su pico entre 1911 y 1920, para luego descender bruscamente. De acuerdo con esto, se observa que el ritmo de ventas de tierras coincide con el ritmo de las exportaciones de lana de ovino, pero como Jacobsen dice es necesario explicar por qué a partir de la década de 1890 este proceso se hace masivo.

Hay dos factores externos que pueden haber incidido en este desarrollo: primero, la guerra civil de inicios de la década de 1890; y segundo el surgimiento de minería aurífera y la explotación de caucho en la caja de selva puneña. Esto provocó que un buen contingente de personas llegara al Altiplano, procedente en su mayoría de Arequipa. Jacobsen observa el registro electoral de la provincia de Azángaro y aprecia que lo 436 ciudadanos inscritos en 1897, 96 (22\%) habían nacido fuera de la provincia. Los que llegaban compraban tierras campesinas con el fin de establecerse como hacendados (Burga y Flores Galindo, 1987). 
ISSN: 2710-0499 ISSN-L: 2710-0480

Jacobsen en el análisis de otros factores, nos da una explicación que consideramos como dialéctica respecto a la transferencia de tierras, pues integra su explicación sobre la base de las relaciones de dependencia personal entre hacendado y campesino. El autor llama a esta relación clientelaje, y la ve relacionada con un cambio en el modo dominante de extracción del excedente económico del campesinado indígena. Este cambio se aprecia como producto de las transformaciones políticas, económicas y sociales acaecidas desde el siglo XVIII.

Esta dialéctica se observa en qué; "En tanto las transferencias de tierras de los campesinos a los hacendados por lo general no se efectuaban como una transacción mercantil inmediata entre agentes económicos libres e iguales, sino que, por el contrario se integraba al posible vendedor al circuito de clientelaje dependiente del hacendado, el período comprendido aproximadamente entre mediados de la década de 1850 y el fin de la primera tendencia de crecimiento en la economía de exportación lanera de la región alrededor de 1867, simplemente puede haber sido demasiado breve como para producir una transferencia masiva de tierras.

De este modo, una parte del creciente número de adquisiciones de tierras hechas por hacendados entre 1890 y 1920 puede entenderse como el efecto acumulativo de las estrategias de expansión iniciadas con bastante anterioridad. En este sentido, cabe destacar el hecho que muchos contratos de venta notariales constituyen únicamente la formalización de una transferencia de una posesión de facto de la tierra, producidas cinco, diez y hasta veinte años atrás" (Jacobsen, 1983b, p. 105).

A esta explicación se refuerza cuando a partir de la década de 1890, se adopta el concepto de tierra como mercancía, no sólo entre los hacendados sino también el campesinado indígena. Este concepto no surge gratuitamente, sino que surge de la propia actividad económica, la cual hizo que aumente el valor de las estancias, debido al crecimiento de la rentabilidad en la cría de ganado, dada el alza en los precios de la lana por la demanda. Todo esto puede haber servido para que los campesinos vendieran sus pastizales.

El precio promedio de las tierras de pastoreo subió un $23.5 \%$, de 161 soles a 199 soles por transacción en el período entre los años de 1899 y la primera década de este siglo. Las tasas de alquiler presentan una evolución cíclica que responde a la demanda de la lana. Durante 1840 y 1850, eran habituales las tasas de alquiler del 10\% sobre el capital ganadero patrimonial. Durante la década de 1860 , debido al primer boom de las exportaciones la tasa de alquiler subió al 15\%. A inicios de la década de 1870 empieza un declive que pone la tasa entre 10 y $12 \%$. Luego hacia 1900 sube a $15 \%$ y entre 1904-1917, gracias al boom de las exportaciones la tasa llega a 20\%. Hubo casos que se pagó hasta el 30\% durante los días de cúspide del boom (Jacobsen 1983b, pp. 105-106).

De acuerdo con Mariátegui se asume que las connotaciones religiosas y ancestrales que revestían cada lote de terreno para los campesinos indígenas, no permitieron que estos vieran con claridad el carácter de mercancía de la tierra (Mariátegui 1992, p. 50-53). Nos damos 
cuenta por esto que era necesaria una presión extraeconómica, que por lo general se traducía en coerción física y la manipulación del poder legal (Calisto 1991, p. 1). Un ejemplo de esta situación nos la da Ciro Alegría en su obra El Mundo es Ancho y Ajeno (Alegría 1997: Cap. VII "Juicios de linderos": 155-187). Además de esto se hacía valer otro tipo de presión que guarda lugar con las relaciones de dependencia personal.

Estas connotaciones religiosas y la no-aplicación del concepto de tierra-mercancía, no significan que el campesino indígena estuviera fuera de la economía de mercado o que no poseyese sus tierras como propiedad privada. Los campesinos eran parte de la economía de mercado ya que, en ella, ellos constituían el papel de productores de materia prima y consumidores de productos manufacturados. Además, esta situación permitió que algunos campesinos ricos compraran tierras a los hacendados con la finalidad de incrementar sus ganancias.

La manipulación legal puede observarse, para la época estudiada en la legislación que desde 1852 busco por un lado enajenar la propiedad rotativa ("moyas", tierra de pastura); y por el otro individualizar la propiedad comunal, para convertir al comunero en pequeño propietario (Valdez de la Torre, 1921, p. 145). Estas propuestas apuntaban a favorecer a los terratenientes, ya que así podría incrementar sus tierras y poder convertir al campesino en yanacona, pongo, huacchileros, etc.

\section{Haciendas ganaderas en el altiplano}

En el departamento de Puno la ganadería fue la principal actividad económica y fue tradicionalmente la fuente de ingreso más importante para la población, sobre todo desde la Independencia, en que esta actividad resulta ser más constante (Appleby, 1979, p. 59). Este departamento presenta características interesantes y además muy peculiares desde comienzos del siglo XIX, y que se reafirmarían hasta la década del 30 del siglo XX.

1.- Los latifundios predominan de manera creciente en las zonas pastoriles, mientras que los minifundios son característicos en las zonas agrícolas que solo es posible en la costa lacustre, densamente pobladas (Escobar, 1967 y Martínez, 1969).

2.- La organización de la producción ganadera se dividió en dos sectores: a) Los hacendados dominaban la producción ovina, y b) los indígenas comuneros la de alpaca (Appleby 1979, p. 59).

3.- A nivel de la estructura agraria y de la explotación agropecuaria se producirán grandes cambios; a) desde la Independencia hasta 1919, expansión de las haciendas y explotación extensiva, y b) de 1920 a 1930, se detiene la expansión y se inicia explotación intensiva (Jacobsen, 1983).

\section{Cuadro No 6}

Numero de ayllus y haciendas en Puno 1876

\begin{tabular}{|c|c|c|c|}
\hline PROVINCIAS & AYLLUS & HACIENDAS & POBLACIÓN \\
\hline AZÁNGARO & 64 & 178 & 46338 \\
\hline CARABAYA & 34 & 0 & 11221 \\
\hline
\end{tabular}

Revista Revoluciones =77- Vol. 3, No 4 (2021), pp. 68-85

Esta obra está bajo una licencia internacional Creative Commons Atribución 4.0. 
ISSN: 2710-0499 ISSN-L: 2710-0480

\begin{tabular}{|c|c|c|c|}
\hline CHUCUITO & 107 & 49 & 42353 \\
\hline HUANCANÉ & 72 & 54 & 45527 \\
\hline PUNO & 121 & 233 & 59480 \\
\hline SANDIA & 33 & 7 & 12721 \\
\hline LAMPA Y AYAVIRI & 74 & 182 & 44811 \\
\hline TOTALES & $\mathbf{5 0 5}$ & $\mathbf{7 0 3}$ & $\mathbf{2 6 2 4 5 1}$ \\
\hline
\end{tabular}

Fuente: Quiroga (1915, pp. 64-67).

\section{Cuadro $\mathrm{N}^{\circ} 7$}

Crecimiento del número de haciendas en Puno

\begin{tabular}{|c|c|c|c|}
\hline PROVINCIAS & $\mathbf{1 8 7 6}$ & $\mathbf{1 9 1 5}$ & $\begin{array}{c}\text { AUMENTO EN 39 } \\
\text { AÑ OS }\end{array}$ \\
\hline AZÁNGARO & 178 & 611 & 433 \\
\hline CARABAYA & 0 & 127 & 127 \\
\hline CHUCUITO & 49 & 242 & 193 \\
\hline HUANCANÉ & 54 & 133 & 79 \\
\hline PUNO & 233 & 854 & 621 \\
\hline SANDIA & 7 & 199 & 192 \\
\hline LAMPAY AYAVIR & 182 & 1536 & 1354 \\
\hline TOTALES & $\mathbf{7 0 3}$ & $\mathbf{3 7 0 2}$ & $\mathbf{2 9 9 9}$ \\
\hline
\end{tabular}

Fuente: Quiroga (1915, p. 69), Gallegos (1924, p. 25) y Romero (1926).

\section{Cuadro No 8}

Haciendas y ganado lanar en Puno 1924

\begin{tabular}{|c|c|c|c|c|}
\hline \multirow{2}{*}{ PROVINCIA } & NÚMERO DE & \multicolumn{3}{|c|}{ TIPO DE GANADO } \\
\cline { 3 - 5 } & HACIENDAS & OVINO & ALPACA & LLAMAS \\
\hline AYAVIRI & 854 & $5^{\prime} 850000$ & 950000 & 450000 \\
\hline AZÁNGARO & 611 & $5^{\prime} 465000$ & 30000 & 88000 \\
\hline CARABAYA & 125 & 405000 & 24000 & 21000 \\
\hline CHUCUITO & 242 & $3^{\prime} 400000$ & $1^{\prime}$ O000000 & 700000 \\
\hline HUANCANÉ & 133 & 460000 & 40000 & 20000 \\
\hline LAMPA & 682 & $5^{\prime} 150000$ & 120000 & 40000 \\
\hline PUNO & 373 & $3^{\prime} 500000$ & 240000 & 150000 \\
\hline SANDIA & 199 & 5000000 & 40000 & 150000 \\
\hline TOTALES & $\mathbf{3 1 8 6}$ & $\mathbf{2 4} 7 \mathbf{7 3 0 0 0}$ & $\mathbf{2} \mathbf{4 4 4 0 0 0}$ & $\mathbf{1}^{\prime} \mathbf{6 1 9 0 0 0}$ \\
\hline
\end{tabular}

Fuente: Gallegos (1924, pp. 27).

Hacia finales del siglo XIX, se puede citar el caso de una pequeña hacienda, la "Hacienda Miraflores”, de Víctor B. Janés, que tenía, para el Primero de Enero de 1899, un capital de 6115 cabezas de ganado ovejuno, las que aumentaran a 6827, hacia el 30 de Junio, según el balance de medio año (Jiménez, 1901, p. 32-62).

Ahora pasaremos a referimos específicamente a la estructura interna, es decir a la organización y administración de la Hacienda, tanto en sus formas de trabajo y las relaciones económicas y sociales que reflejan las haciendas de esta zona del país, durante el boom de las exportaciones de la lana de ovino.

El ritmo de la expansión de las haciendas hasta 1920 aproximadamente se ve como "respuesta a la tendencia a largo plazo del alza de los precios de la lana"; resulta ser evidente si se considera el régimen laboral y la conducción económica de las haciendas ganaderas durante 
este período. En vez de invertir capital en las operaciones ganaderas y optimizar así las ganancias con una producción intensiva, los hacendados optaron por incrementar la producción lanera expandiendo sus operaciones y el número de trabajadores arrendatarios, pero sin alterar la naturaleza extensiva y tradicional de las operaciones ganaderas (Jacobsen, 1983b, p. 107). Es decir, el incremento de una ganadería extensiva permitió la subsistencia de la base económica (Caballero, 1969; y Matos Mar y Mejía, 1980).

La fuerza de trabajo fundamental de las haciendas ganaderas fueron los colonos o yanaconas, que eran una especie de trabajadores arrendatarios. Este tipo de trabajador no solo participaba en faenas como las propias a la ganadería y a la agricultura sino también cumplían actividades domésticas y transportaba los productos de las haciendas al mercado con sus propios animales, y además asumía otras obligaciones.

Sobre las formas de trabajo en las haciendas hay diversidad de planteamientos:

a. Unos dicen que yanaconaje y colonato son similares o iguales (Jacobsen 1983b, p. 107).

b. Otros dicen que yanaconaje y aparcería son similares o iguales (Comisión para la Reforma Agraria en el Perú. Exposición de motivos y Proyecto de Ley, 1960, p. 46).

c. Pero nosotros nos inclinamos a creer que yanaconaje es una forma propia y distinta del colonato y aparcería (Parain, Vilar y otros 1985, pp. 45-47).

Antes de todo intentaremos definir y diferenciar a grandes rasgos estas formas de trabajo y tenencia de tierra. El yanacona recibía una parcela del hacendado y se comprometía con ello a trabajar para él y era "parte de un sistema de servidumbre con variantes según la actividad principal -agrícola o ganadera- y las características sociales y culturales regionales" (Matos Mar, 1976, p. 37).

Este tipo de trabajo era común en la sierra del Perú. En la costa se practicó, pero con variantes que le hacen similares a otras formas de trabajo como el colonato, pero a pesar de las apariencias, "el colono era fundamentalmente un peón asalariado de la hacienda que, a semejanza, del yanacona, recibía una parcela para su propio cultivo. En cambio, el yanacona no participaba de la explotación directa de la hacienda; aunque esporádicamente fuera peón trabajaba personalmente la extensión que recibía en arriendo, cumpliendo en oportunidades obligaciones de tipo servil" (Matos Mar 1976, p. 32). El colonato se aprecia como actividad dominante en las haciendas azucareras y arroceras.

La aparcería se distingue del colonato y yanaconaje ya que "era un simple arrendamiento de tierras a cambio de una renta en producto, cuya proporción variaba según el cultivo y la zona" (Matos Mar, 1976, p. 36). Además, presenta otra característica, que es además el rasgo más importante de su funcionamiento, y es que el propietario hacendado compartía con el aparcero el riesgo de la producción, lo que no sucedía en el yanaconaje, en el que la renta era fija (Matos Mar, 1976, p. 37). Las compensaciones que recibían los colonos a cambio de trabajo pueden dividirse en dos partes, el "ingreso campesino" y el "ingreso salarial". El primero era el derecho de usufructuar los pastos y la tierra de cultivo de la hacienda y el segundo era el pago en especie o/y dinero que hacia la administración de la hacienda. Los 
ISSN: 2710-0499 ISSN-L: 2710-0480

pastores recibían generalmente un salario en forma de víveres que eran llamados "avíos", de maíz, quinua, charqui, coca, alcohol (Martínez Alier, 1984, p.13).

Con estas características "el tipo de desarrollo que podría haberse esperado en el régimen laboral de las haciendas ganaderas del Altiplano como respuesta de la tendencia de crecimiento a largo plazo de las exportaciones laneras, y particularmente durante el boom de la primera guerra mundial, sería de un marcado intento de los propietarios de haciendas por abandonar un sistema de mano de obra arrendataria que se pagaba principalmente en derechos de usufructo, por un sistema de mano de obra asalariada" (Jacobsen, 1983b, p. 108). Esto es lo que se podría suponer si no se observa la importancia que los derechos de usufructo de las tierras pastorales y agrícolas de la hacienda tenían en el ingreso total de los colonos, ya que era más conveniente tanto para hacendados y colonos mantener esa forma de ingreso. Además, los aumentos salariales producidos de 1840 hasta 1920 no fueron suficientes para modificar el equilibrio entre el ingreso de los colonos como campesinos y su ingreso salarial, en favor de una porción preponderante del ingreso derivado de los salarios en el ingreso total de los colonos, esto es, para hacer de ellos trabajadores asalariados en esencia.

Se aprecia en el caso de Azángaro, que los hacendados intentaron imponer como una norma "el aumento de la fuerza de trabajo permanente en sus estancias, de forma paralela a la expansión de su base de tierras entre 1860 y 1920" (Jacobsen, 1983b, p. 109). Esto tuvo gran éxito ya que cuando el campesino indígena vendía sus tierras, en el contrato se comprometía a trabajar y a servir con lealtad como colono o yanacona. Esto se verificó cuando se observan los censos generales de población de 1876 y 1940, la porción de la población rural de Azángaro que vivía permanentemente en haciendas paso de un $23.4 \%$ a un $35.8 \%$. Más de 21 mil habitantes pasaron a las haciendas durante esos años.

Esto nos permite observar tres factores principales que señalaron el carácter extensivo y tradicional de la crianza de ovinos en el Altiplano. El primero es la degeneración de los animales, en términos genéticos, ya que no había una continua renovación de la especie, que se traduciría en la incorporación de animales de raza pura a los rebaños. Esto último se hizo pero con poca difusión. La segunda es la casi total ausencia de cercos. La mayoría de las haciendas del Altiplano eran unidades de producción que funcionaban con pastizales de régimen abierto. La ausencia de cercos no permitía el control del ganado, con respecto al contacto con los animales 'chuscos' y enfermos, y a los robos.

La tercera era la confiabilidad absoluta en los pastos naturales, habiendo siempre el peligro de la escasez y la sequía. Esto hacía dependiente la producción con respecto a las circunstancias climáticas y estacionales. Esto hizo imposible mantener el capital ganadero en las haciendas en equilibrio constante con las cantidades de forraje disponibles. Los hacendados no compraban forraje, en tiempos de sequía, se limitaban a reservar una parte de los pastos. Hay otros factores para dar una naturaleza extensiva y tradicional a la cría de ovinos del Altiplano. Como la alta tasa de mortalidad que se aprecia en Azángaro en inicios de siglo, en un 5 y $10 \%$ del total de ovejas y más de 50\% de borregos recién nacidos. Pudo ser

Revista Revoluciones -80- Vol. 3, No 4 (2021), pp. 68-85 Esta obra está bajo una licencia internacional Creative Commons Atribución 4.0. 
causada por la falta de forraje que mantenía débil al animal y propenso a cualquier enfermedad, y además a las inclemencias climáticas (Jacobsen, 1983b, pp. 110-111).

Las soluciones a la alta tasa de mortalidad, fueron diversas; unos plantearon e incrementaron el capital en ganado, ya que teniendo más ganado en especial más ovejas fértiles se podría tener mayor cantidad de borregos y que mayor cantidad sobreviva. Otros plantearon y maximizaron el capital en ganado en sus haciendas a pesar de una baja productividad por animal. Debía de haber dos pariciones al año por cada oveja, a pesar que esta constancia debilitaba a las madres y hacia correr riesgo de muerte de ella y el borrego, además por estar débil la madre no podía alimentar al borrego, y este moría. Otro de los factores que contribuye a este tipo de ganadería extensiva, es la escasa y aislada utilización de innovaciones técnicas en la cría y cuidado de los ovinos, así como en la esquila y el cuidado de la lana.

Hubo hacendados que introdujeron innovaciones técnicas pero estas permanecieron demasiado aisladas como para conducir a un cambio cualitativo en la conducción económica de la crianza de ganado. Jacobsen nos dice algo, que consideramos determinante, cuando se refiere a los factores que caracterizan como extensiva y tradicional a la cría de ovinos. Para este autor "el cambio en una zona particular de la estructura interna de las haciendas no podía mantenerse sino se emprendía un cambio sistemático de las relaciones de producción”. Esto se explica ya que "la introducción de carneros padres de pura raza con fines de reproducción no podía conducir a mejoras de base más amplias en la raza de las ovejas, que constituían el capital ganadero de una estancia, a no ser que se abandone el sistema de pastoreo conjunto del ganado de la hacienda y del pastor, construyendo cercos. La construcción de corrales no podía producir un impacto significativo en los índices de mortalidad del ganado, salvo que se adoptasen medidas para superar la escasez de forraje" (Jacobsen, 1983b, pp. 112-113).

En Azángaro estos cambios o intentos sistemáticos casi no se produjeron antes de 1920, ni siquiera durante los años del boom de las exportaciones laneras, de 1914-1919. Pero como el sistema de crianza y comercialización del ganado en el sur peruano logró responder a los ciclos de corto plazo y las tendencias a largo plazo en la demanda de lana en los mercados internacionales. Aquí este autor llega a plantear unas hipótesis de las probables causas del incremento de la actividad ganadera.

La primera gran hipótesis, es que la población del Altiplano atravesó por una tendencia de crecimiento a largo plazo a través del período republicano hasta los años de 1960. Esta hipótesis se basa en los supuestos: a) entre 1780 y 1820 o hasta 1850 , las unidades ganaderas se vieron diezmadas como consecuencia de los frecuentes conflictos bélicos y guerras civiles. Luego de estos años se presenció probablemente la recuperación natural de la población ganadera. b) la crianza de ganado proporcionaba la fuente principal de ingresos a la mayoría de los pobladores altiplánicos", y el incremento de 250 mil personas en 1850 a 550 mil el 1940 aproximadamente, no podía haberse producido sin un incremento en la población ganadera. 
ISSN: 2710-0499 ISSN-L: 2710-0480

De acuerdo a las fuentes que se tienen no se puede precisar las cifras exactas para dar consistencia a estas hipótesis, pero si se observa el cálculo de las tasas anuales de extracción y reposición, del Plan Regional para el Desarrollo de Sur del Perú llegó, en 1859, a una cifra de crecimiento anual de $4 \%$ para la población ovina del departamento de Puno (3\% para la provincia de Azángaro). Estas cifras no pueden hacerse extensivas durante el período, desde el siglo XIX al XX, pero demuestran que hay una tendencia de crecimiento en cantidad de ovinos a largo plazo. "En este sentido, entonces, el aumento de las poblaciones ganaderas permitió a los productores de lana del Altiplano responder a las tendencias de largo plazo elevando los precios de lana en el mercado internacional entre mediados del siglo XIX y 1872, y aproximadamente entre 1896 y 1918/19" (Jacobsen, 1983b, pp. 113-114).

Podemos preguntarnos, siguiendo a Jacobsen: ¿̇y qué puede decirse en relación a las fluctuaciones cíclicas a corto plazo? ¿Cómo respondió entre ellas la economía del sur peruano? Aquí se observa el papel de las estrategias de las casas comerciales, de comprar para almacenar (Burga y Reátegui 1981). Además, cuando se preveía un aumento en los precios internacionales, se comenzaba a almacenar lana y cuando se producían descensos bruscos en los precios, se llegaba incluso a limitar o suspender las compras y las ventas de lana. Así tanto los campesinos indígenas como los hacendados ajustaban el volumen de venta de lana a las condiciones del mercado.

Como resumen Jacobsen planteó que "la economía rural del Altiplano se adaptó de dos maneras a la tendencia a largo plazo de la creciente demanda de lana iniciada en la década de 1890 y que culminará con el boom de la Primera Guerra Mundial. La cantidad de producción lanera atravesó por una tendencia de crecimiento a largo plazo que fue el resultado de la recuperación natural de las poblaciones ganaderas. En el corto plazo, los ajustes en las exportaciones laneras ante la fluctuación de la demanda se basaron principalmente en estrategias de precios, almacenamiento y esquileo, trazadas por los comerciantes y productores de lana. Pero más importante para el desarrollo posterior de la sociedad altiplánica fue el cambio masivo en la distribución de la producción agregada de lana entre el sector campesino y el de los propietarios, como resultado de la incorporación de muchas tierras y de un gran número de campesinos en las haciendas antiguas y recién creadas" (Jacobsen, 1983b, p. 115).

Este planteamiento resulta importante en la medida en que se considera en el largo plazo el proceso de cambio entre la distribución agregada de la producción de lana entre los campesinos libres y la hacienda, como resultado de: a) del aumento del número de haciendas y la incorporación de más tierra a las antiguas; y b) del incremento de la mano de obra en las haciendas. Aquel planteamiento, puede compararse con los de otros autores (Burga, Flores Galindo, Reátegui, Bonilla y Martínez Alier), y a nuestro parecer resulta ser más objetivo y completo, desde la perspectiva del análisis económico, pero se hace necesario analizar otros factores, como los sociales y políticos que confluyen en este gran fenómeno que es "el desarrollo del subdesarrollo" del sur del Perú (Appleby, 1979, p. 55). 


\section{CONCLUSIONES}

No es exagerado decir que las fluctuaciones de la economía de exportación lanera, produjeron malestar entre los campesinos. Entre 1866 e inicios de 1868, en Azángaro y Huancané, hubo levantamientos y protestas campesinas, conocidas como la rebelión de Bustamante, que tienen relación con la lucha política entre facciones gamonales de la región que apoyaban indistintamente las candidaturas de Prado o Diez Canseco a la presidencia, y al parecer se oponían únicamente al restablecimiento de la contribución personal y de los abusos específicos de las autoridades locales.

Estos levantamientos ocurrieron durante una de las cúspides de la exportación, además ponen de manifiesto la existencia de tendencias reivindicativas opuestas a la usurpación de tierras de los campesinos, cosa que había aumentado durante esos años. Además es probable una relación entre el intento de reimposición del impuesto individual a los indígenas y la lucha contra esto, en el momento de ingresos máximos por la venta de lanas. Pero no es coincidencia que en esta época de altas ganancias, el episcopado de Puno decidiera completar la construcción de la catedral, obligando a cada indio a pagar una 'limosna' de dos pesos para tal fin (Jacobsen, 1993).

El impacto de las fluctuaciones sobre el malestar campesino se hace más evidente desde la década de 1890, y tiene relación con el ciclo de crecimiento que va de esta década hasta 1918/19. Desde la década de 1890 los levantamientos fueron provocados por diversas acciones contra del campesinado indígena, como: el impuesto a la sal; el intento de exigir de nuevo el impuesto individual; los abusos por parte de gobernadores y curas; y la usurpación de las tierras de los campesinos (Kapsoli, 1987, pp. 26-34). Estas luchas comenzaron a basarse cada vez más en el problema de la tierra (Zúniga, 1989). Enfrentamientos como los de Chupa, Arapa y Samán entre 1909 y 1913, y la llamada rebelión de Rumi Maqui de 1915 y 1916 en San José (Vasallo, 1978, pp. 123-127). Fueron originados mayormente por la posesión de los pastizales (Paredes, 1976, p. 264; Jacobsen, 1983b, p. 116).

Jacobsen nos dice que es necesario para este tipo de trabajos, saber si estas rebeliones se deben a los "métodos más rapaces de concentración de tierras por parte de los hacendados o de rebeliones más activas por parte de los campesinos de la comunidad" (Jacobsen, 1983b, p. 116). Este es un problema para la Historia que básicamente debe de buscar descubrir si hubo o no desarrollo de la conciencia social campesina o de una conciencia de clase, y a la vez como se manifiesta en términos de reconocimiento, organización y una práctica común como clase.

Para este autor lo importante para su estudio es que se ve que todos los sectores o "grupos sociales cuyas bases económicas residían en la crianza de ganado estaban dispuestas a valerse de métodos más activos para salvaguardar sus intereses conforme los riesgos aumentaban al subir rápidamente los precios de la lana". Para este autor en el boom de las exportaciones los campesinos indígenas, a pesar de los abusos, obtuvieron alguna "ganancia derivada del creciente valor de su producción lanera. Esta combinación paradójica de intensos abusos e ingresos crecientes aumentó la disposición del campesino del Altiplano para defender (y, en

Revista Revoluciones -83- Vol. 3, No 4 (2021), pp. 68-85

Esta obra está bajo una licencia internacional Creative Commons Atribución 4.0. 
ISSN: 2710-0499 ISSN-L: 2710-0480

lo posible, ampliar) su 'porción de la torta' bajo condiciones de un 'juego de suma cero', en el cual, a largo plazo, sólo la distribución, pero no el nivel absoluto de los ingresos, podía variar" (Jacobsen, 1983b, pp. 116-117).

Este planteamiento nos presenta una nueva interpretación, en la que el campesinado indígena no solo se defiende del abuso del gamonal y sus aliados (autoridades y curas), sino también defiende lo que gana y puede ganar, de los demás agentes económicos que tienen como el campesino su base económica en la cría de ganado para la producción lanera. Los abusos contra el campesinado y los intereses económicos de este, resultan como dos caras de la misma moneda, cosa que en última instancia tomará el camino de la rebelión, es decir, la lucha contra los abusos para el mantenimiento de sus intereses económicos, frente, a los gamonales.

\section{REFERENCIAS BIBLIOGRÁFICAS}

Bonilla, H. (1975) Gran Bretaña y el Perú 1826-1919; informes de los Cónsules Británicos. (5 tomos). Lima: IEP.

Comisión para la Reforma Agraria y la Vivienda (1960) La Reforma Agraria en el Perú. Exposición de motivos y Proyecto de Ley. Lima: Impreso en los talleres Gráficos P.L. Villanueva S.A.

Gallegos, E. (1924) Estudio económico del departamento de Puno. Tesis de Bachiller en Derecho. Arequipa: UNSA.

Jiménez, V. A. (1901) Breves apuntes sobre la contabilidad de las haciendas de ganadería, en el departamento de Puno. Puno: Imprenta de "La Segunda Época".

Quiroga, M. A. (1915) La Evolución jurídica de la Propiedad Rural en Puno. Tesis de Doctorado en Jurisprudencia. Arequipa: Universidad de Arequipa. Tipografía Quiroz Perea.

Salas Perea, G. (1966) Monografia sintética de Azángaro. Puno: Editorial Los Andes.

Romero, E. (1926) Monografia del Departamento de Puno. Lima: Imprenta Torres Aguirre.

Valdez de la Torre, C. (1921) Evolución de las Comunidades indígenas. Lima: Editorial Euforión.

\section{BIBLIOGRAFÍA}

Alegría, C. (1997) El mundo es ancho y ajeno. Lima: Editorial Mantaro.

Appleby, G. (1979) “Las transformaciones del sistema de mercados en Puno: 1890-1960”. Análisis No 8-9. Lima.

Basadre, J. (1968-69) Historia de la República 1822-1933. Tomos IX-X. $6^{\text {a }}$ edición corregida y aumentada. Lima: Editorial Universitaria S.A.

Bertram, G. (1977) "Modernización y cambio en la industria lanera en el sur del Perú, 1919-1930: un caso de desarrollo frustrado". Apuntes Vol. III, № 6. Lima.

Bonilla, H. (1974) "Islay y la economía del sur peruano en el siglo XIX". Apuntes $\mathrm{N}^{\circ}$ 2. Lima.

Bourricaud, F. (1963) "Castas y clases en Puno". Revista del Museo Nacional, Tomo xxxii. Lima.

Burga, M. y Flores Galindo, A. (1987) Apogeo y crisis de la República Aristocrática. Lima: Ediciones Rikchay Perú.

Burga, M. y Reategui, W. (1981) Lanas y capital mercantil en el sur. La casa Ricketts, 1895-1935. Lima: IEP.

Caballero, J. M. (1981) Economía agraria de la sierra peruana. Antes de la Reforma Agraria de 1969. Lima: IEP.

Revista Revoluciones -84- Vol. 3, No 4 (2021), pp. 68-85

Esta obra está bajo una licencia internacional Creative Commons Atribución 4.0. 
Calisto, M. (1991) “Campesinos puneños y resistencia cotidiana. 1900 -1930”. Allpanchis, año XXIII, $\mathrm{N}^{\circ} 37$. Cusco

Chevalier, F. (1966) "Témoignages litteraires et disparités de croissance: l'expansion de la grande propiété dans le Haut-Pérou au XXe siécle”. Annales E.S.C., Vol. XXI. Paris.

Escobar, G. (1967) Organización social y cultural del sur del Perú. México: Instituto Indigenista Interamericano.

Flores Galindo, A. (1977) Arequipa y el sur andino, siglos VXIII-XX. Lima: Editorial Horizonte.

Flores Galindo, A., Plaza, O. y Ore, T. (1978) “Oligarquía y capital comercial en el sur peruano (18701930)". Debates en Sociología No 3. Lima.

Flores Ochoa, J. (1983) "Pastoreo de llamas y alpacas en los Andes -balance bibliográfico-”. Revista Andina, Año 1, t. 1, setiembre. Cuzco.

Jacobsen, N. (1983) Landtenure and society in the Peruvian Altiplano: Azángaro province, 17701920. (2 t.) Tesis Dr. University of California. 1982.

Jacobsen, N. (1983b) “Ciclos y booms en la agricultura de exportación latinoamericana: El caso de la economía ganadera en el Sur peruano, 1855 1920”. ALLPANCHIS año XIII, vol. XVIII, No 21. Cusco.

Jacobsen, N. (1993) Mirages of Transition: The Peruvian Altiplano, 1780-1930. Berkeley: University of California Press.

Jacobsen, N. (2013) Ilusiones de la transición: El altiplano peruano, 1780-1930. Lima: Instituto de Estudios Peruano.

Kapsoli, W. (1982) "Memorial del indio puneño 1900-1930". Tarea, o 6 1982. Lima.

Kapsoli, W. (1987) Los Movimientos Campesinos en el Perú. Lima: Ediciones Atusparia.

Mariátegui, J. C. (1992) 7 Ensayos de interpretación de la realidad peruana. Lima: Editorial Horizonte.

Martínez, H. (1969) Las migraciones altiplánicas y la colonización del Tambopata. Lima: Centro de Estudios Población y Desarrollo.

Martinez Alier, J. (1974) Los Huacchilleros del Perú. Dos estudios de formaciones sociales agrarias. Lima: IEP-Ruedo Ibérico.

Matos Mar, J. (1976) Yanaconaje y Reforma Agraria en el Perú. El caso del valle del Chancay. Lima: IEP.

Matos Mar, J. y Mejia, J. (1980) La Reforma Agraria del Perú. Lima: IEP.

Paredes A., M. (1976) “El Levantamiento Campesino de "Rumi-Maqui”. En: Visión de las Ciencias Histórico-sociales (Tomo II) de Fernando Lecaros. Lima: Retablo de papel.

Parain, C., Pierre Vilar y otros (1985) El Feudalismo. Madrid: SARPE.

Spalding, K. (1977) “Estructura de clases en la sierra peruana, 1750-1920”. Análisis № 1. Lima.

Tamayo Herrera, J. (1982) Historia social e indigenismo en el Altiplano. Lima: Ediciones Treintaitres.

Vasallo, M. (1978) "Rumi-Maqui y la nacionalidad quechua”. ALLPANCHIS, Vol. XI-XII. Cusco.

Zuñiga, J. (1989) Azángaro: Camino y efectos del Decreto Ley 17716 en su Estructura Agraria. Balance y Proyección de una Tesis. Lima: CONCYTEC. 